\title{
On the Use of Quantum Thermal Bath in Unimolecular Fragmentation Simulations
}

\author{
Riccardo Spezia*,† and Hichem Dammak ${ }^{\ddagger}$ \\ †Laboratoire de Chimie Théorique, Sorbonne Université and CNRS, F-75005 Paris, France \\ $\ddagger$ Laboratoire Structures Propriétés et Modélisation des Solides, CentraleSupélec, CNRS, \\ Université Paris-Saclay, F 91190 Gif-sur-Yvette, France \\ E-mail: riccardo.spezia@sorbonne-universite.fr
}




\begin{abstract}
In the present work we have investigated the possibility of using the Quantum Thermal Bath (QTB) method in molecular simulations of unimolecular dissociation processes. Notably, QTB is aimed in introducing quantum nuclear effects with a computational time which is basically the same as in newtonian simulations. At this end we have considered the model fragmentation of $\mathrm{CH}_{4}$ for which an analytical function is present in the literature. Moreover, based on the same model a microcanonical algorithm which monitor zero-point energy of products, and eventually modifies trajectories, was recently proposed. We have thus compared classical and quantum rate constant with these different models. QTB seems to correctly reproduce some quantum features, in particular the difference between classical and quantum activation energies, making it a promising method to study unimolecular fragmentation of much complex systems with molecular simulations. The role of QTB thermostat on rotational degrees of freedom is also analyzed and discussed.
\end{abstract}

July 12, 2019

\title{
Introduction
}

Unimolecular dissociation represents one of the elementary chemical process which is involved in a series of phenomena, like e.g. collisional activation fragmentation or laser induced reactivity. ${ }^{1}$ The kinetics of this process is described by a simple exponential decay of the initial microcanonical ensemble ${ }^{2} N(0)$ :

$$
\frac{N(t)}{N(0)}=e^{-k t}=e^{-t / \tau}
$$

where $k$ is the unimolecular rate constant and $\tau$ the lifetime. The well-known RiceRamsperger-Kassel-Marcus (RRKM) statistical theory is often employed to describe this 
process, ${ }^{1,3,4}$ also called Quasi-Equilibrium theory (QET). ${ }^{5-7}$ In this framework, the initial ensemble decays with a single exponential behavior and the rate constant, $k$, is the RRKM rate constant. If information on reactants and transition states are available, it is possible to obtain both $k(E)$ and $k(T)$ by means of analytical models. Analytical theories are surely very powerful, but have limited applications: one has to determine partition functions (or density of states) of reactants and transition states and all the possible pathways should be known in advance. They often employ harmonic approximations: anharmonicity can be added but this becomes almost impossible for relatively large molecules. Explicit simulations have been used and developed to directly obtain unimolecular fragmentation dynamics and products. ${ }^{8-11}$ At this aim, chemical dynamics simulations were used with different ways of energizing the fragmenting molecule: ${ }^{12}$ (i) giving an excess of internal energy, (ii) by explicit collision with an inert gas. For example, it was possible to understand products structures and reaction mechanisms in collision induced dissociation (CID) of several systems, from small organic molecules, ${ }^{13-15}$ to biological molecules like peptides ${ }^{16-20}$ or sugars. ${ }^{21,22}$

In addition to information on fragmentation products and mechanisms, chemical dynamics can be used to obtain kinetic informations on unimolecular dissociation. In particular, from time decay of the initial population it is possible to obtain rate constants ${ }^{9,10,16,18}$ and in some case threshold energies, via a correspondence between classical microcanonical RRKM expression (called also RRK theory) and temperature dependence of rate constant which assumes an Arrhenius-like form. These dynamics are purely newtonian, and thus rate constants are classical and anharmonic - the anharmonicity comes directly since the simulations are done on-the-fly on the full potential energy surface which is not harmonic. Quantum nuclear effects are not considered and this can impact the simulations outcome for (at least) three aspects: (i) the rate constant is classical; (ii) the activation or threshold energies will not consider zero-point energy (ZPE) difference between reactant and transition state (and/or products); (iii) tunneling is not considered. Furthermore, there can be in principle a difference between classical and quantum dynamics in reaction product ratio for the 
fragmentation of a complex molecule, with an effect on product abundances and even on appearance of some of them.

In endothermic unimolecular dissociation an important quantum nuclear effect is related to the ZPE of the products. In classical simulations, in fact, it is possible to form products with vibrational energy smaller than their ZPE, which is not allowed in quantum dynamics.

To recover at least some of these quantum nuclear effects, a number of methodologies were proposed in last years. Methods based on path integral molecular dynamics, like ring polymer MD (RPMD) ${ }^{23}$ or centroid MD (CMD) ${ }^{24}$ can provide ZPE conservation. ${ }^{25,26}$ They are based on path integral theory ${ }^{27,28}$ and thus well designed for thermodynamic properties. ${ }^{29}$ They are also used for dynamical quantities but they must be carefully handled. ${ }^{30}$ Computationally, they increase the simulation time by a factor $P$ with respect to a classical trajectory, where $P$ is the number of beads which represents each particle in the path-integral formalism.

Semi-classical methods ${ }^{31}$ can overcome most of the problems related to incorrect treatment of nuclear quantum effects in trajectories, like was shown recently in the case of HermanKluk $^{32}$ propagator, ${ }^{33}$ but they need a huge statistics to converge and are rarely applicable to systems with more than three degrees of freedom. More in general, in last years, a number of methods were proposed to specifically avoid ZPE leakage, often based on the knowledge of normal modes and projection of actual positions and momenta on internal coordinates. ${ }^{34-37}$

Recently, Dammak and co-workers have proposed a method called Quantum Thermal Bath $(\mathrm{QTB})^{38}$ which can recover some nuclear quantum effects of vibrational motion. This method was mainly tested for condensed phase systems. ${ }^{39-44}$ It is based on a Langevindynamics formalism with a colored noise (which keeps quantum vibrational properties) and computationally it has almost the same cost as a newtonian dynamics. Furthermore, it can be applied to molecular dynamics propagation without any need of specific knowledge of ZPE or instantaneous normal modes. Being promising for its use in a range of problems, its applicability to model chemical reactions was never investigated.

In the present work, we study the case of a model unimolecular reaction, notably the 
fragmentation of $\mathrm{CH}_{4}$. Recently, the same system was studied by Paul and Hase ${ }^{45}$ by comparing purely classical fragmentation trajectories with trajectories in which the ZPE energy of the forming products was checked and trajectories modified if they do not have enough ZPE. Following the spirit of original Miller-Hase-Darling method ${ }^{46}$ for constraining ZPE in non-reactive systems, in the present case if a trajectory is leaving the reactant basin without enough ZPE in the product (here $\mathrm{CH}_{3}$ ) it is sent back to reactants and "another chance" to react with correct ZPE energy is given. We have thus used the same model fragmentation, to understand how QTB can include nuclear quantum effects in modeling unimolecular reactivity. Furthermore, we have investigated how, from microcanonical simulations at different energies, the Paul and Hase correction to trajectories impacts the energy evolution of rate constants and thus of activation energies. Finally, using well-known relationship connecting $k(E)$ and $k(T)$ it was possible to compare the different approaches on the same reaction.

\section{Models and Methods}

\section{Model for $\mathrm{CH}_{4} \rightarrow \mathrm{CH}_{3}+\mathrm{H}$ dissociation}

We have employed the well-studied Duchovic-Hase-Schelegel (DHS) model ${ }^{47}$ for $\mathrm{CH}_{4}$ fragmentation with later modifications. ${ }^{48,49}$ It is composed by a set of Morse functions for the $\mathrm{C}-\mathrm{H}$ bonds plus additional torsional potential to keep the tetrahedral structure. Details of the model are given in the Appendix. Note that another model for $\mathrm{CH}_{4}$ is present in the literature, ${ }^{50,51}$ but here we used the same modified-DHS model employed by Paul and Hase in order to be comparable with this recent study. ${ }^{45}$ The aim of the present work is merely to understand QTB performances in unimolecular dissociation, in order to eventually use it for other more complex systems.

Within this potential, the $\mathrm{ZPE}$ of the reactant, $\mathrm{CH}_{4}$, is $29.17 \mathrm{kcal} / \mathrm{mol}$, and that of the $\mathrm{CH}_{3}$ product is $18.60 \mathrm{kcal} / \mathrm{mol}$. The classical fragmentation barrier is $109.46 \mathrm{kcal} / \mathrm{mol}$, while, taking into account ZPE of reactant and products, the barrier is expected to drop down to 
$98.89 \mathrm{kcal} / \mathrm{mol}$.

This model was recently used by Paul and Hase to investigate the microcanonical dissociation kinetics using a purely classical dynamics with a new algorithm which keeps the system back to reactant if the products $\left(\mathrm{CH}_{3}\right)$ are obtained without enough $\mathrm{ZPE} .{ }^{45}$ In this

work, the $\mathrm{C}-\mathrm{H}$ distance of $6 \AA$ was fixed as the value to define when a dissociation is done (irreversibly). This value was chosen as in Ref. ${ }^{45}$ such that we can directly compare results for both canonical and microcanonical simulations.

Within this model, and the energies employed here, the unimolecular fragmentation reaction allowed to occur is:

$$
\mathrm{CH}_{4}^{*} \rightarrow \mathrm{CH}_{3}+\mathrm{H}
$$

Using the harmonic frequencies of the DHS model for $\mathrm{CH}_{4}$, the average classical, $E^{c l}$, and quantum, $E^{q}$, vibrational energies as a function of the temperature, $T$, can be calculated from the classical statistical mechanics and Bose-Einstein statistics, respectively:

$$
\begin{aligned}
\left\langle E^{c l}\right\rangle & =N k_{B} T \\
\left\langle E^{q}\right\rangle & =\sum_{i}^{N} \hbar \omega_{i}\left(\frac{1}{2}+\frac{1}{e^{\beta \hbar \omega_{i}}-1}\right)
\end{aligned}
$$

where $N$ is the number of vibrational frequencies, $k_{B}$ is the Boltzmann constant, $\omega_{i}$ are the vibrational angular frequencies, $\hbar$ is the reduced Planck's constant and $\beta=1 / k_{B} T$. The temperature dependence of classical and quantum average vibrational energies for the present $\mathrm{CH}_{4}$ model are reported in Figure 1.

\section{Quantum Thermal Bath Simulations}

Molecular dynamics simulations were done using the Quantum Thermal Bath (QTB) method to take into account quantum nuclear effects. ${ }^{38}$ The Langevin formal structure of equations 


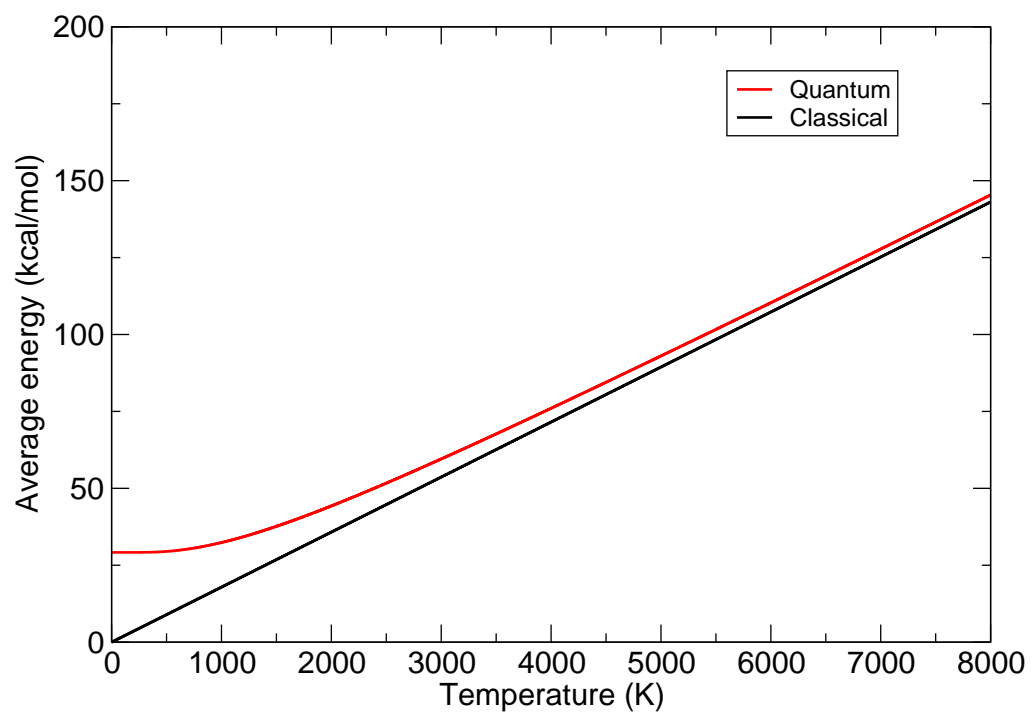

Figure 1: Quantum versus classical average vibrational energy of the $\mathrm{CH}_{4}$ model employed as a function of temperature.

of motion is used

$$
m_{i} \ddot{q}_{i, \alpha}=-\frac{\partial V}{\partial q_{i, \alpha}}-m_{i} \gamma \dot{q}_{i, \alpha}+R_{i, \alpha}(t)
$$

where $i$ runs on the atoms, $\alpha=x, y, z, m_{i}$ is the mass, $\dot{q}_{i, \alpha}$ and $\ddot{q}_{i, \alpha}$ are the first and second time derivatives of the positions, $V$ the interaction potential between the nuclei (here the modified DHS described previously), $\gamma$ an effective frictional coefficient and $R_{i, \alpha}(t)$ is the random force which in QTB method is set in order to have the correct spectral density, $I_{R}$, following the Wiener-Khinchin theorem

$$
\left\langle R_{i, \alpha}(t) R_{i, \alpha}(t+\tau)\right\rangle=\int_{-\infty}^{+\infty} I_{R_{i}}(|\omega|) e^{-i \omega \tau} \frac{d \omega}{2 \pi}
$$

where $I_{R_{i}}$ obeys to the fluctuation-dissipation theorem for quantum systems: ${ }^{52}$

$$
I_{R_{i}}(\omega)=2 m_{i} \gamma \hbar \omega\left[\frac{1}{2}+\frac{1}{\exp (\beta \hbar \omega)-1}\right]
$$


The random force was generated as reported previously ${ }^{53,54}$ before each simulation and then equations of motion integrated with modified velocity Verlet algorithm with a time step of 0.1 fs. Different $\gamma$ values were employed and results studied as a function of this parameter.

Six temperatures were considered in fragmentation simulations: 3000, 3500, 4000, 4500, 5000 and $5500 \mathrm{~K}$. For each set of simulations, 500 trajectories were done, with variable length (between 5 ps and $1 \mathrm{~ns}$ ) as a function of temperature. These time-lengths were chosen in order to have almost $100 \%$ of reactive trajectories. Simulations were done under two regimes: (i) the action of the bath was removed from translational motion; (ii) the action of the bath was removed from both rotational and translational motion.

All simulations were performed using Venus chemical dynamics software ${ }^{55}$ which was modified to introduce integration of Langevin equations of motion reading externally provided random forces.

\section{Langevin Molecular Dynamics Simulations}

Langevin Molecular Dynamics (LMD) simulations were performed analogously to QTB ones. The structure of equations of motion are the same as Eq 5, where now the random force is the white noise, corresponding to the classical spectral density:

$$
I_{R_{i}}=2 m_{i} \gamma k_{B} T
$$

and the simulations were done as previously, with the same algorithms where now a different random force is read. The same temperatures and $\gamma$ values of QTB simulations were considered in LMD ones. As previously, 500 trajectories with variable time-lengths (between 5 ps and $4 \mathrm{~ns}$ ) as function of temperature were performed per each set of simulations. As for QTB, simulations were done under two regimes: (i) the action of the bath was removed from translational motion; (ii) the action of the bath was removed from both rotational and 
translational motion. The LMD dynamics was implemented in Venus as for QTB, reading an external noise.

\section{Microcanonical Dynamics}

Microcanonical simulations were done as in Ref, ${ }^{45}$ extending the study at different internal energies: 119.9, 127.8, 131, 133, 136.6, 145.35, 154.16, 162.98 and $171.82 \mathrm{kcal} / \mathrm{mol}$. For these energies, we run trajectories that are purely newtonian and also using the reversing momentum algorithm of Paul and Hase. ${ }^{45}$ Briefly, the reversing momentum algorithm (here and hereafter called REV) works as follows: a newtonian simulation is performed but when the products are formed (identified in the present case by the $\mathrm{C}-\mathrm{H}$ distance of $6 \AA$ ) the vibrational energy, $E_{v i b}$, of products $\left(\right.$ here $\mathrm{CH}_{3}$ ) is calculated. If $E_{v i b} \geq \mathrm{ZPE}$ (in this case the $\mathrm{ZPE}$ of $\mathrm{CH}_{3}$ product) then the trajectory is considered reactive and stopped. If, otherwise, the products have not enough $\mathrm{ZPE}$, the momentum of $\mathrm{CH}_{3}-\mathrm{H}$ relative motion is reversed, corresponding to sending back the trajectory to the reactant basin. The trajectory follows its faith and if again at a certain point it reacts the $\mathrm{E}_{v i b}$ of $\mathrm{CH}_{3}$ is checked again, up to fragmentation with the correct $\mathrm{E}_{v i b}$. More details can be found in the original paper by Paul and Hase. ${ }^{45}$ Note that initially only the vibrational degrees of freedom are activated, which is the microcanonical counterpart of regime (ii) in QTB and LMD simulations (i.e. when the action of the bath is removed also from rotational degrees of freedom).

Also in this case, we performed 500 trajectories for each energy value and method with variable time-lengths (between 500 ps and $100 \mathrm{~ns}$ ) in order to have almost $100 \%$ of reactive trajectories. 


\section{Results}

\section{Canonical lifetimes}

From QTB and LMD simulations it is possible to obtain properties in the canonical ensemble and thus, if we measure the lifetime of the reactants, their canonical lifetime, $\tau(T)$, and corresponding rate constants, $k(T)(k(T)=1 / \tau(T))$. In Figure 2 we show the population decay obtained at two temperatures for QTB simulations with $\gamma=0.1 \times 10^{14} \mathrm{~s}^{-1}$. They can be fitted with an exponential function and obtain $\tau$ and $k$. Similar exponential decay is obtained for QTB and LMD simulations at different $\gamma$ and temperature values.
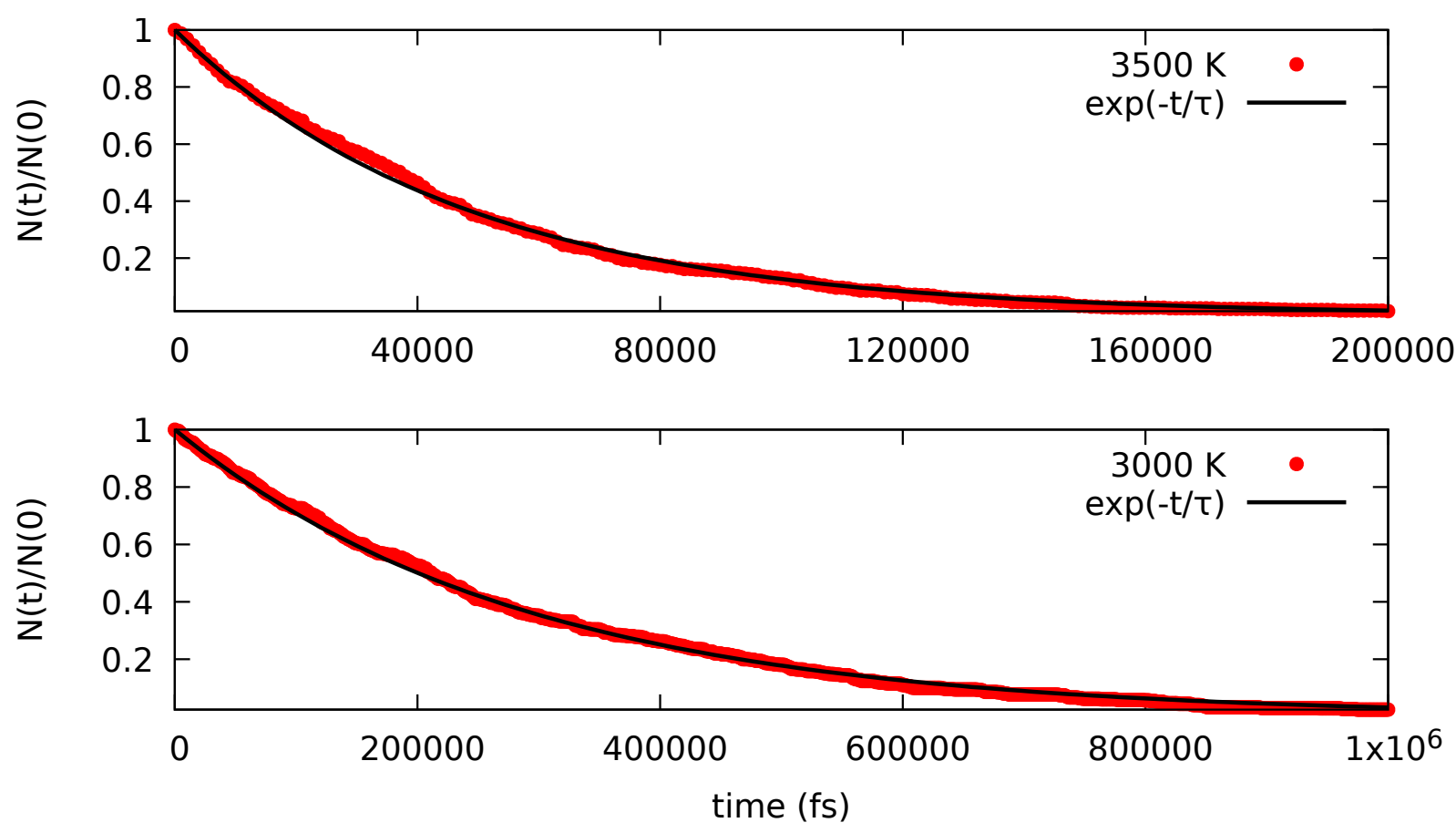

Figure 2: $\mathrm{CH}_{4}$ population decay as obtained in QTB simulations for $\gamma=0.1 \times 10^{14} \mathrm{~s}^{-1}$ at two different temperatures.

One crucial aspect of QTB and LMD simulations is the choice of the frictional parameter, $\gamma .1 / \gamma$ represents the characteristic time of energy exchange between the system and the thermostat and it is usually chosen higher than the life-time of vibrational modes in order to avoid widening of peaks and bands in frequency spectra. In QTB its role is more subtle. 
In fact, the QTB method is prone to ZPE leakage (ZPEL) problem which results from the transfer of energy from high-frequency to low-frequency modes. Recently, Brieuc et al. ${ }^{54}$ have shown that increasing the $\gamma$ value reduces and even eliminates the ZPEL. A more detailed study of Mangaud et al. showed that it is possible to adapt $\gamma$ on-the-fly in order to fulfill the quantum fluctuation dissipation theorem: ${ }^{56}$ this approach is surely tempting but needs equilibrated portion of trajectories (to calculate power spectra) so it cannot be directly used for relatively fast reactivity. However, high $\gamma$ values are not possible since the bath frequency should not be in resonance with the vibrational frequencies. We have thus chosen the intermediate temperature of $4000 \mathrm{~K}$ to understand how $\tau$ is affected by the $\gamma$ parameter. Furthermore, the coupling with rotational degrees of freedom can further increase the width, so we have performed tests at $4000 \mathrm{~K}$ also removing the bath on rotational motion. In Figure 3 we report the lifetimes for both QTB and LMD with $\gamma$ in the range $0.01-0.5 \times 10^{14} \mathrm{~s}^{-1}$. We notice that, for $\gamma$ being in the $0.1-0.5 \times 10^{14} \mathrm{~s}^{-1}$, lifetime values are relatively constant. We have thus considered this range for further analysis. Removing the bath also from rotational motion slows down the reaction, both in QTB and LMD simulations. This is quite expected since the rovibrational coupling increases the reactivity which here consists in simple dissociation of $\mathrm{H}$ atom. The effect of $\gamma$ is the same as previously, confirming that the $0.1-0.5 \times 10^{14} \mathrm{~s}^{-1} \gamma$ range provides constant lifetimes (and thus rate constants).

QTB and LMD lifetimes at different temperatures and $\gamma$ values are summarized in Table 1. As we can noticed, QTB lifetimes are systematically smaller than the LMD ones at the same temperature: this is not unexpected since for the same formal temperature the classical and quantum internal energies are different (see Figure 1). This is not in contradiction with the previous microcanonical results where using REV algorithm (which considers ZPE in products) the lifetime increases with respect to classical trajectories: quantum distribution in QTB is in both reactants and products such that the effective barrier decreases when quantum nuclear effects are considered. On the other hand, REV algorithm controls only 


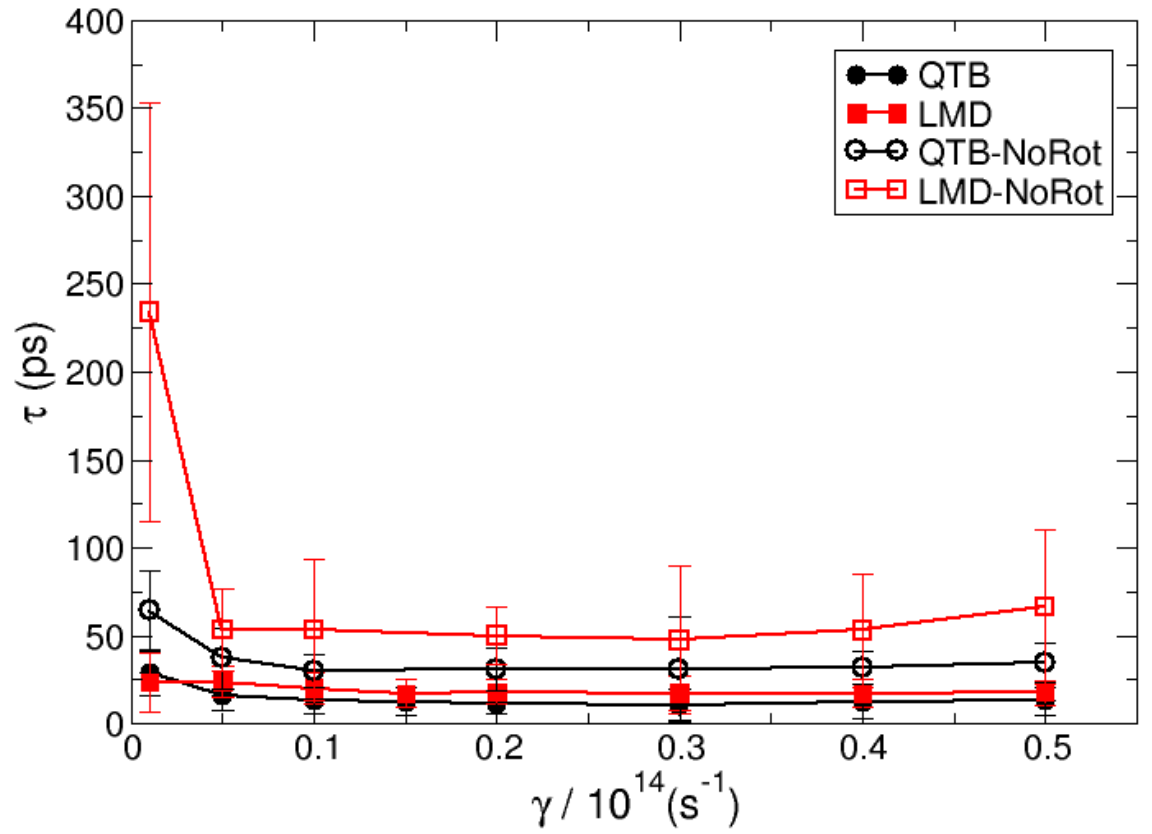

Figure 3: Canonical lifetimes for $\mathrm{T}=4000 \mathrm{~K}$ as a function of $\gamma$ as obtained in QTB and LMD simulations. Filled symbols are for simulations where the bath was removed only on translational degrees of freedom, open ones where it was removed also on rotational motion (labeled NoRot). 
the ZPE of products.

\section{Activation Energies}

Canonical simulations, both LMD and QTB, show temperature dependences of the rate constant which follow an Arrhenius behavior for all the $\gamma$ values considered here. An example, as obtained for $\gamma=0.2 \times 10^{14} \mathrm{~s}^{-1}$, is reported in Figure 4 .

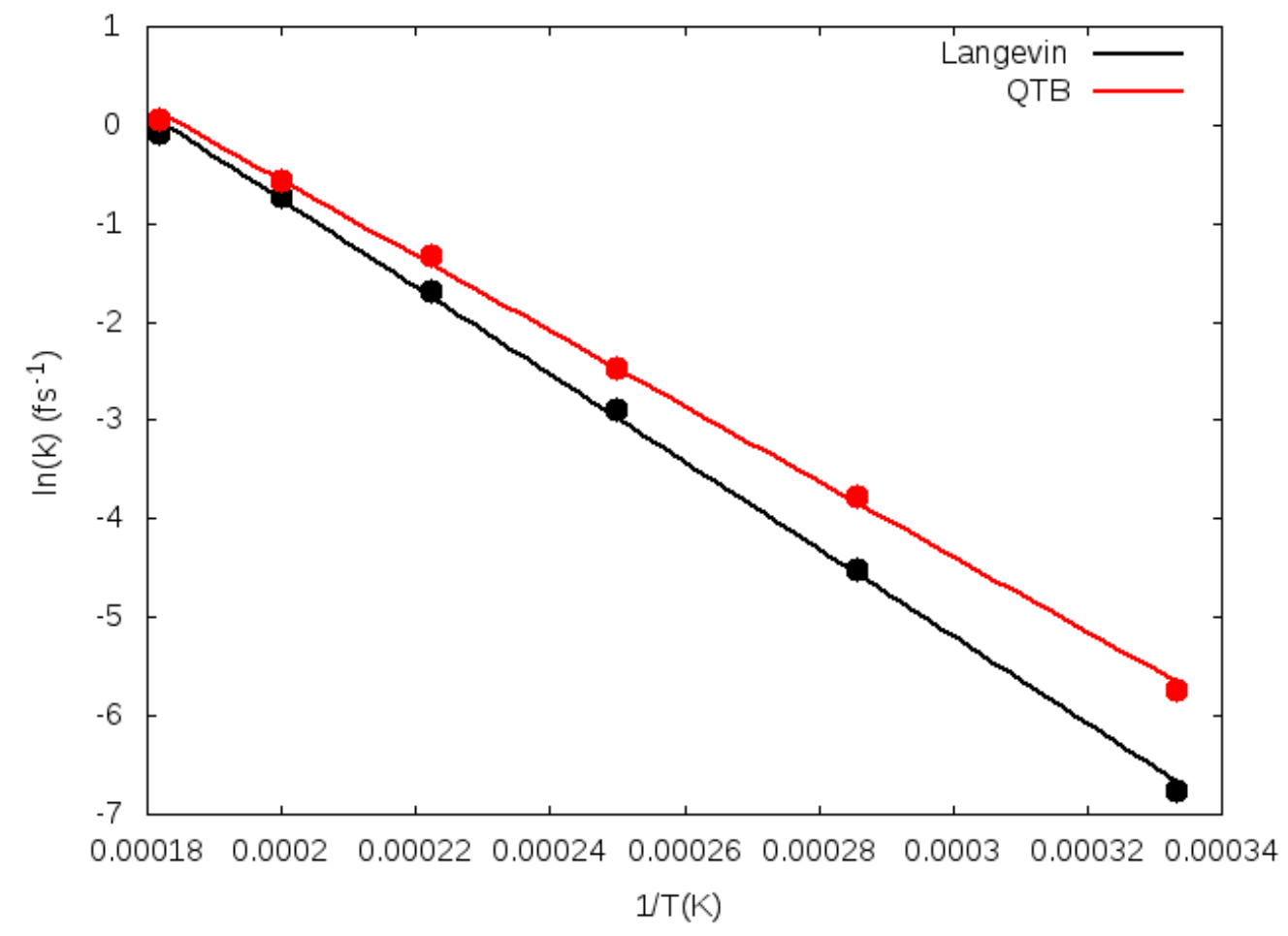

Figure 4: Arrhenius plot for $\gamma=0.2 \times 10^{14} \mathrm{~s}^{-1}$ as obtained from LMD and QTB simulation.

We have thus fitted $k(T)$ with the well-known expression:

$$
k(T)=A e^{-E_{a} / k_{B} T}
$$

where $k_{B}$ is the Boltzmann constant, obtaining the pre-exponential factor, $A$, and the activation energy, $E_{a}$, for LMD and QTB simulations at different $\gamma$ values. Activation energy and pre-exponential factor values are reported in Table 2. 
Table 1: Fragmentation lifetimes (in ps) as obtained in QTB and LMD (also when the bath was removed from rotation, labeled as NoRot) at different $\gamma$ values.

\begin{tabular}{|c|c|c|c|c|c|}
\hline $\mathrm{T} / \mathrm{K}$ & $\gamma / 10^{14} \mathrm{~s}^{-1}$ & QTB & QTB-NoRot & LMD & LMD-NoRot \\
\hline \multirow{5}{*}{3000} & 0.1 & 289.11 & 757.62 & 937.34 & 3126.37 \\
\hline & 0.2 & 307.73 & 843.29 & 855.31 & 3189.67 \\
\hline & 0.3 & 281.76 & 916.53 & 941.34 & 3493.33 \\
\hline & 0.4 & 332.86 & 1146.12 & 999.15 & 3670.72 \\
\hline & 0.5 & 347.77 & 1142.34 & 1171.00 & 4107.19 \\
\hline \multirow[t]{5}{*}{$\overline{3500}$} & 0.1 & 48.40 & 111.28 & 93.85 & 272.35 \\
\hline & 0.2 & 43.64 & 121.33 & 91.92 & 286.80 \\
\hline & 0.3 & $44 . .55$ & 163.54 & 95.63 & 266.16 \\
\hline & 0.4 & 50.63 & 139.74 & 89.11 & 325.99 \\
\hline & 0.5 & 54.94 & 167.77 & 104.51 & 389.54 \\
\hline \multirow[t]{5}{*}{4000} & 0.1 & 13.04 & 30.14 & 20.25 & 53.58 \\
\hline & 0.2 & 11.74 & 31.04 & 18.10 & 50.13 \\
\hline & 0.3 & 10.64 & 31.31 & 17.33 & 47.49 \\
\hline & 0.4 & 12.13 & 31.85 & 17.08 & 53.00 \\
\hline & 0.5 & 12.82 & 34.82 & 17.73 & 66.26 \\
\hline \multirow[t]{5}{*}{4500} & 0.1 & 4.45 & 9.81 & 5.97 & 14.44 \\
\hline & 0.2 & 3.78 & 8.74 & 5.40 & 13.61 \\
\hline & 0.3 & 3.56 & 9.74 & 4.96 & 13.62 \\
\hline & 0.4 & 4.09 & 9.78 & 4.64 & 14.89 \\
\hline & 0.5 & 3.61 & 11.61 & 5.19 & 15.04 \\
\hline \multirow[t]{5}{*}{5000} & 0.1 & 2.23 & 4.62 & 2.71 & 5.51 \\
\hline & 0.2 & 1.77 & 3.80 & 2.08 & 5.01 \\
\hline & 0.3 & 1.61 & 3.51 & 1.99 & 4.78 \\
\hline & 0.4 & 1.57 & 3.99 & 1.87 & 4.92 \\
\hline & 0.5 & 1.63 & 4.71 & 1.92 & 4.98 \\
\hline \multirow[t]{5}{*}{$\overline{5500}$} & 0.1 & 1.20 & 2.38 & 1.40 & 2.88 \\
\hline & 0.2 & 0.95 & 2.00 & 1.08 & 2.30 \\
\hline & 0.3 & 0.86 & 1.89 & 0.99 & 2.18 \\
\hline & 0.4 & 0.83 & 2.00 & 0.97 & 2.29 \\
\hline & 0.5 & 0.84 & 2.00 & 1.19 & 2.34 \\
\hline
\end{tabular}


Table 2: Activation energies (in $\mathrm{kcal} / \mathrm{mol}$ ) and pre-exponential factors (in $\mathrm{ps}^{-1}$ ) as obtained from QTB and LMD simulations by fitting Eq. 9. In parenthesis we report results when removing the bath also on rotational degrees of freedom.

\begin{tabular}{lcccc}
\hline$\gamma$ & $\mathrm{E}_{a}^{Q T B}$ & $\mathrm{E}_{a}^{L M D}$ & $\ln \mathrm{A}^{\text {QTB }}$ & $\ln \mathrm{A}^{L M D}$ \\
\hline 0.1 & $72 \pm 1$ & $85 \pm 2$ & $6.5 \pm 0.1$ & $7.6 \pm 0.3$ \\
& $(75 \pm 1)$ & $(92 \pm 1)$ & $(6.1 \pm 0.1)$ & $(7.5 \pm 0.3)$ \\
0.2 & $76 \pm 1$ & $88 \pm 1$ & $7.1 \pm 0.1$ & $8.1 \pm 0.2$ \\
& $(78 \pm 2)$ & $(95 \pm 2)$ & $(6.5 \pm 0.2)$ & $(7.9 \pm 0.3)$ \\
0.3 & $77 \pm 1$ & $90 \pm 2$ & $7.2 \pm 0.1$ & $8.4 \pm 0.3$ \\
& $(83 \pm 2)$ & $(96 \pm 2)$ & $(7.0 \pm 0.2)$ & $(8.1 \pm 0.2)$ \\
0.4 & $79 \pm 1$ & $91 \pm 2$ & $7.6 \pm 0.1$ & $8.6 \pm 0.3$ \\
& $(83 \pm 1)$ & $(97 \pm 2)$ & $(7.0 \pm 0.1)$ & $(8.2 \pm 0.2)$ \\
0.5 & $80 \pm 1$ & $93 \pm 2$ & $7.6 \pm 0.1$ & $8.7 \pm 0.3$ \\
& $(83 \pm 1)$ & $(99 \pm 2)$ & $(6.8 \pm 0.1)$ & $(8.3 \pm 0.2)$ \\
\hline
\end{tabular}

We should note, first, that QTB activation energies are systematically lower than corresponding LMD ones. Furthermore, $E_{a}$ increases with $\gamma$ for both QTB and LMD. It is well known that activation energies obtained from Arrhenius plot of a canonical simulation are often lower than actual potential energy barriers, due to the energy fluctuations in a canonical ensemble. ${ }^{57,58}$ Interestingly, here, the QTB values are lower than those of LMD as should be from differences in ZPE between reactants and products. In particular, from the $\mathrm{CH}_{4}$ model employed, we will expect a difference between classical and quantum energy barrier of $10.57 \mathrm{kcal} / \mathrm{mol}$ : the differences between LMD and QTB activation energies are plot in Figure 5 at different $\gamma$ values and they are very similar (and less dependent on $\gamma$ ) to the reference value.

We should notice that $\Delta E_{a}$ values obtained when removing the thermostat effect on both rotational and translational motions are systematically higher than those for which the thermostat was removed only on translational motion. In particular this reflects the larger difference between the two regimes in LMD simulations, as shown in Table 2. 


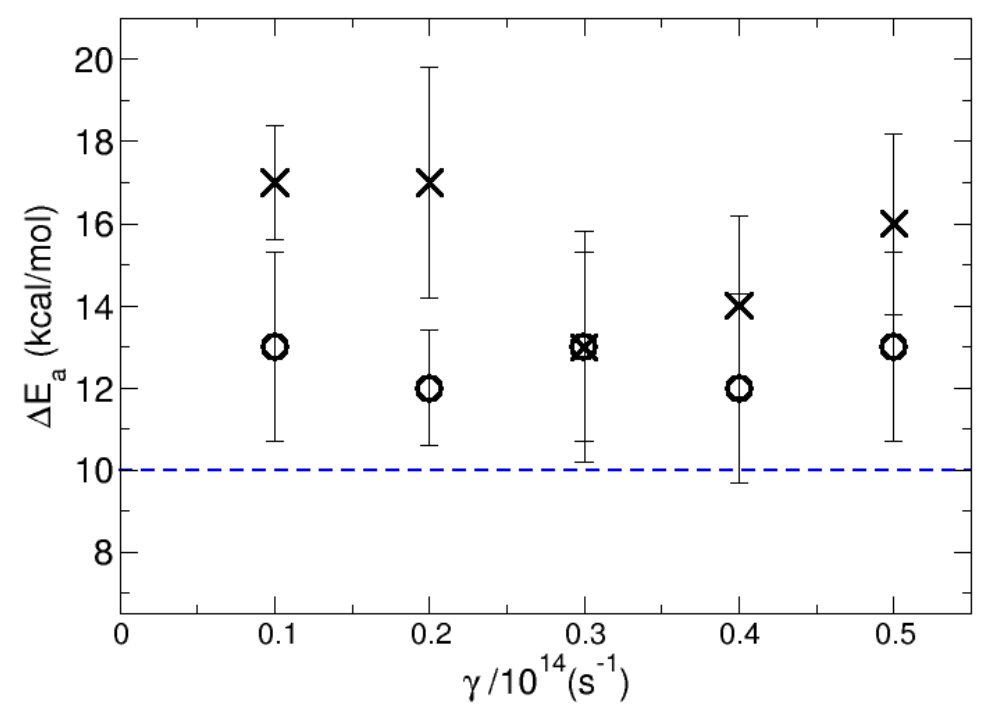

Figure 5: Differences between LMD and QTB activation energies as a function of $\gamma$. Circles correspond to simulations where the thermostat was removed only on translational motion, crosses where also rotational motion was removed. As horizontal line we report the classicalquantum difference of reaction barrier as from the potential energy surface.

\section{Microcanonical lifetimes and barriers}

Microcanonical simulations were done at fixed internal energies, and, as previously, lifetimes were obtained as function of this energy. Note that values for two energies (131 and $133 \mathrm{kcal} / \mathrm{mol}$ ) correspond to results of Ref. ${ }^{45}$ As expected, REV lifetimes are bigger than those from newtonian trajectories, because the REV algorithm has the final effect of extending the time spent by a trajectory in the reactant basin.

We can extract information on reaction barriers from evolution of rate constant as a function of internal energy. Here, the classical RRKM expression can be used since underlying trajectories follow classical equations of motions (in REV algorithm there is only a condition on when considering a trajectory done, if not fullfilled the trajectory is simply sent back to reactants). In particular, data can be fitted using the well-known RRK formula:

$$
k(E)=\nu\left(\frac{E-E_{0}}{E}\right)^{s-1}
$$


where $E_{0}$ is the barrier and $s$ the number of vibrational modes of reactants. In old RRK theory, $\nu$ is an adjustable parameter with the dimension of a frequency. Here we fit the expression of Eq. 10 to get $E_{0}$ and $\nu$, which is an effective frequency.

Other than using the pure RRK relation where the number of degrees of freedom $(s)$ was fixed (such that $s-1=8$ ) we also let this as a free parameter. As shown in Figure 6, this improves the rate constant fit. Resulting parameters are reported in Table 3.
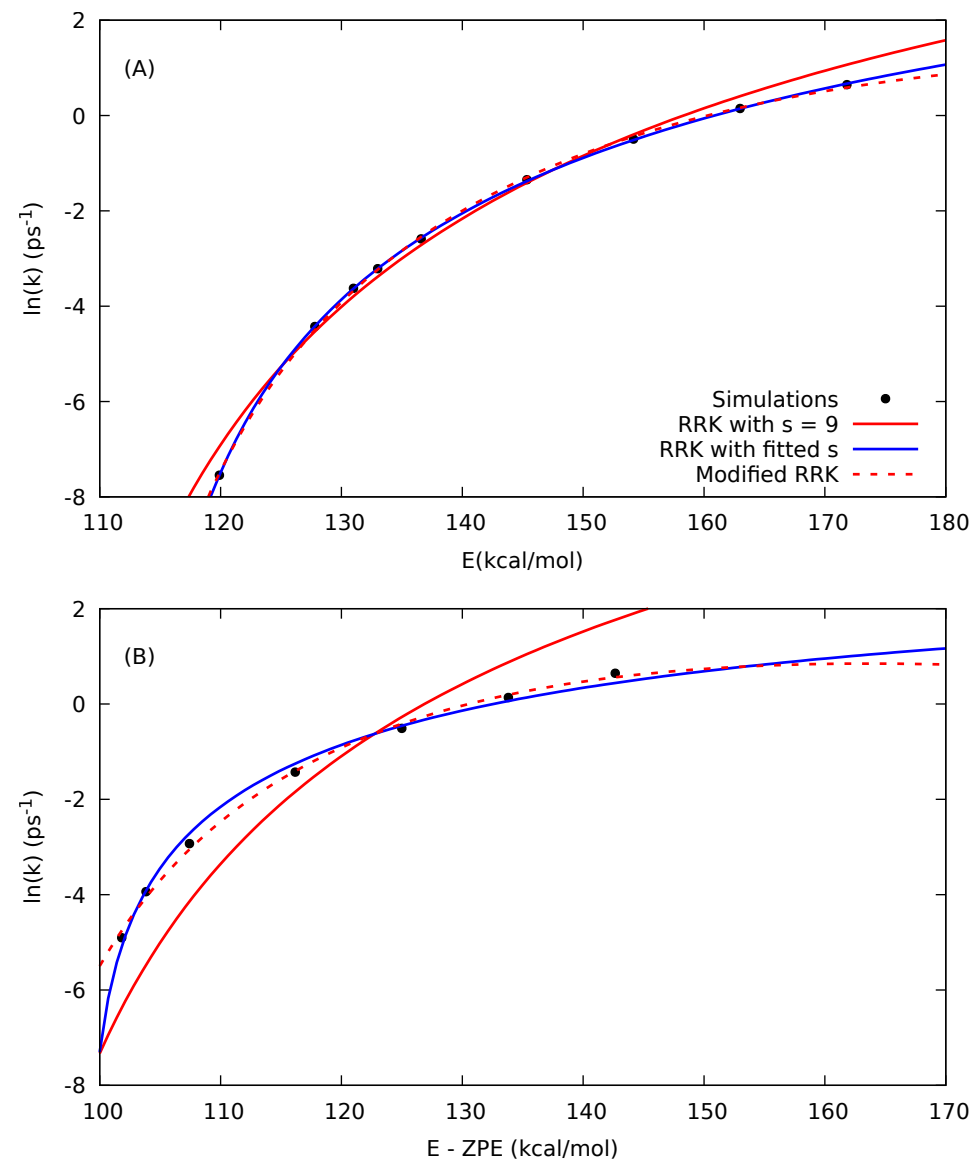

Figure 6: Rate constant as a function of internal energy as obtained in microcanonical simulations. (A) Newtonian simulations; (B) REV algorithm simulations.

The best fits are obtained when $s$ value was added as a parameter, resulting in $s$ values which are systematically lower than the number of degrees of freedom of the system. This is a well-known issue of RRK fits and it is related to the problem of using a classical harmonic rate constant to fit a (classical) anharmonic fragmentation kinetic, as deeply discussed by Song 
Table 3: Energy barriers obtained and other RRK fit parameters obtained from micro-canonical simulations, both using Newton and REV dynamics. Results using Eq 10 are reported in the higher part of the table and those using Eq 11, for which $s$ is fixed to the actual vibrational degrees of freedom, in the lower part. $\Delta$ is the difference (in kcal/mol) between Newton and REV barriers $\left(E_{0}\right)$.

\begin{tabular}{ll|rrrr}
\hline Simulation & $\mathrm{E}_{0}(\mathrm{kcal} / \mathrm{mol})$ & $\nu\left(\mathrm{ps}^{-1}\right)$ & $(\mathrm{s}-1)$ & $\Delta$ \\
\hline Newton & 102 & 3904 & 8 & - \\
Newton & 112 & 338 & 4.9 & - \\
REV & 88 & 11929 & 8 & -14 \\
REV & 99 & 25 & 2.34 & -13 \\
\hline \hline & $\mathrm{D}_{0}(\mathrm{kcal} / \mathrm{mol})$ & $a\left(\mathrm{ps}^{-1}\right)$ & $b\left([\mathrm{kcal} / \mathrm{mol}]^{-1}\right)$ & $\mathrm{c}$ & $\Delta$ \\
\hline Newton & 107 & $2.19 \cdot 10^{6}$ & $3.44 \cdot 10^{-2}$ & 0.02 & - \\
REV & 86 & $4.76 \cdot 10^{6}$ & $5.31 \cdot 10^{-2}$ & 0.06 & 21 \\
\hline \hline
\end{tabular}

and Hase some years ago. ${ }^{59}$ Furthermore, one should consider the energy dependent behavior of the threshold in the case of unimolecular decomposition. The same authors proposed a modified expression for the RRK rate constant, where now frequency and threshold energy are energy dependent:

$$
k(E)=\nu(E)\left[\frac{E-E_{0}(E)}{E}\right]^{s-1}
$$

The energy dependence of the threshold can be expressed as:

$$
E_{0}(E)=D_{0}-c E_{\infty}
$$

where $D_{0}$ is the dissociation energy and $E_{\infty}=E-D_{0}$.

The $\nu(E)$ takes into account (i) the energy dependence in the anharmonic correction ratio between sum and density of states and (ii) the tightens in variational transition state vibrational frequencies with increasing $E$. A simple expression was proposed by Song and Hase: 59

$$
\nu(E)=a f_{a n h}(E)
$$


For $f_{\text {anh }}(E)$ different forms are possible, Song and Hase have found that for $\mathrm{CH}_{4}$ dissociation the best way is using

$$
f_{a n h}(E)=\frac{\exp \left[b^{\dagger}\left(E-E_{0}\right)\right]}{\exp (b E)\left[1+\frac{b E}{s}\right]}
$$

where $b$ is for reactants and $b^{\dagger}$ for transition state.

The modified RRK expression of Eq. 11 depends now only on four fitted parameters, $a, b$, $b^{\dagger}$ and $c$ if one knows $D_{0}$. The fitted values for newtonian dynamics are reported in Table 3 . As in the original work, ${ }^{59} b^{\dagger}=0$ permits a good fit.

When fixing $D_{0}$ to the known classical threshold, $a, b$ and $c$ values are similar to the one reported for the same reaction with a slightly different model. ${ }^{59}$ When letting $D_{0}$ varying as a free parameter, it slightly changes but more importantly other parameters change largely, in particular $a$ and $c$.

In the case of REV simulations, it was not possible to provide a fit with a physical meaning when fixing $D_{0}$ to the known value. Considering $D_{0}$ as an additional fitted quantity (and the ZPE of reactants), we have two slightly similar values (about $86 \mathrm{kcal} / \mathrm{mol}$ ) for both $b^{\dagger}=0$ and $b^{\dagger} \neq 0$. The threshold is now $20 \mathrm{kcal} / \mathrm{mol}$ lower than the classical one and $10 \mathrm{kcal} / \mathrm{mol}$ too low than when adding ZPE in reactant and products. One reason can reside in the arbitrary modification in dynamics when doing REV simulations, resulting in irregular behavior of unimolecular fragmentation kinetics. Another possible reason is that the modified RRK approach is not appropriate to model unimolecular dissociation with REV method. Finally, other anharmonicity functions exist, in particular based on works of Troe, ${ }^{60,61}$ and it would be interesting to explore them deeper in details in works focusing on microcanonical theories, which is, however, beyond the aim of the present paper.

Concluding, microcanonical simulations, in particular with using standard RRK expression, can recover the quantum vs classical energy difference. Barrier values are similar to what expected from the analytical potential in particular if also the number of degrees of freedom is considered as a fit variable. 


\section{Classical vs Quantum rate constants}

Direct comparison between rate constants obtained from LMD and QTB and Newtonian and REV simulations cannot be done because the former lifetimes (and rate constant) are canonical while the latter microcanonical. From Arrhenius and RRK fits it was possible to extrapolate activation and threshold energies which can be compared. In particular, as we have discussed, simulations report that barriers from QTB and REV are lower than those from LMD and Newtonian simulations as expected and the differences are similar to what expected from the energy profile of the reaction.

A more detailed comparison can be done by calculating the canonical rate constant, $k(T)$, from the micro-canonical one, $k(E)$, using the well known relationship:

$$
k(T)=\frac{1}{Q(T)} \int k(E) \rho(E) e^{-\beta E} d E
$$

where $Q(T)$ is the partition function (here only vibrational motion was considered to be coherent with microcanonical initial conditions), $\rho(E)$ the density of states and $\beta=1 / k_{B} T$.

In Figure 7A we compare Newtonian microcanonical simulations with LMD ones for different $\gamma$ values and where the thermostat was removed from both translational and rotational motions (labeled NoRot) and only from translational one. Rate constant using integrated Newtonian microcanonical values are very close to LMD-NoRot values. In panel B of the same Figure 7 we report REV integrated rate constant compared with QTB values. In this case REV rate constant is systematically lower than QTB values and in any case closer to QTB-NoRot values. As we discussed previously, the REV dynamics is biased and it is not clear how (and if) kinetics theory is applicable.

Overall the comparison shows that QTB and REV simulations provide similar results and that QTB is a promising way of using direct dynamics simulations to estimate unimolecular rate constants. 

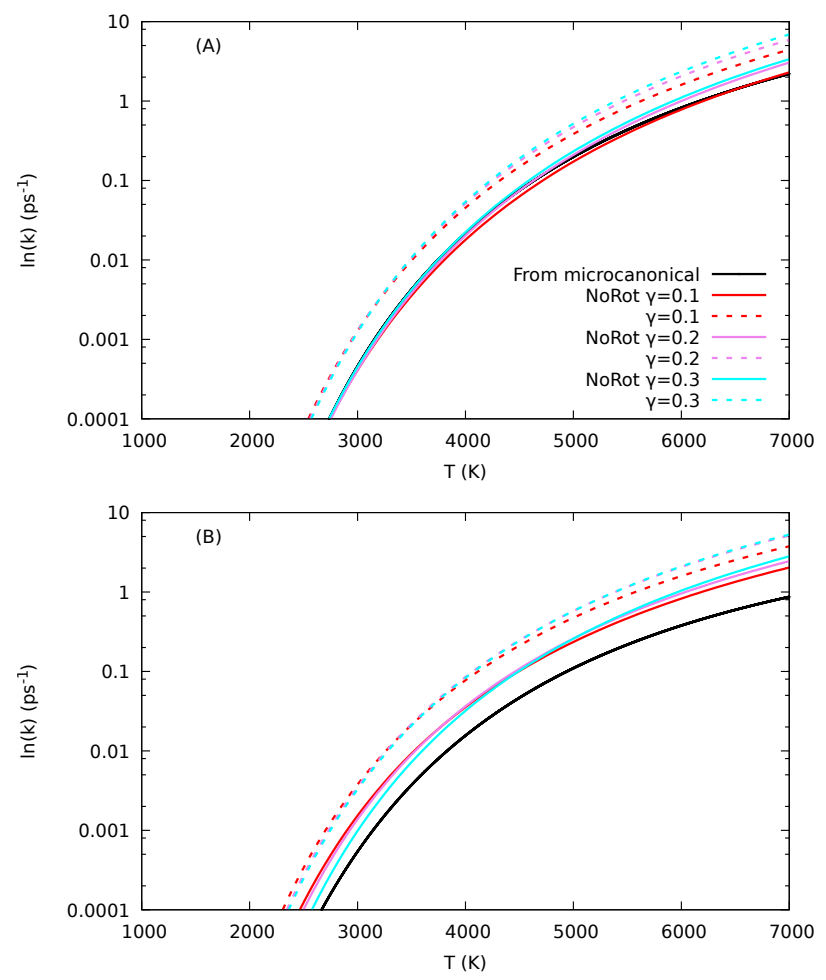

Figure 7: Rate constant as a function of temperature as obtained by canonical simulations at different $\gamma$ values and from integrated microcanonical rate constants using Eq. 15. Panel A: newtonian and LMD simulation results; panel B: REV and QTB simulation results. Different $\gamma$ values are shown in both LMD and QTB simulations. NoRot means that the thermostat (LMD or QTB) is removed also from rotational degrees of freedom. 


\section{Conclusions}

In the present work we have investigated the possibility of using the Quantum Thermal Bath method to include quantum nuclear effects in unimolecular dissociation trajectory simulations. In particular, we were interested in the problem of rate constant modification due to difference in products and reactants vibrational zero-point energies. At this aim we have considered the fragmentation of a model $\mathrm{CH}_{4}$ for which microcanonical algorithms were developed in order to take into account this specific problem. QTB was shown to being able to catch the key feature of the difference between classical and quantum kinetics, i.e. a difference in activation energy of about $10 \mathrm{kcal} / \mathrm{mol}$, with values similar to modified microcanonical algorithm. In this last microcanonical method, it is necessary to know in advance the ZPE of both reactants and products to obtain the correct behavior. The advantage of QTB is that results are directly obtained from simulations without any pre-knowledge of ZPE, because the algorithm is tailed in order to fulfill at best the quantum fluctuation dissipation relationship.

Note that QTB has the computational advantage of being comparable in simulation time with a standard newtonian dynamics, thus making this approach promising for studying direct reaction dynamics of more complex molecular systems. One critical parameter of QTB is the friction value which was subject to careful analysis in the present work. Surely more studies are needed to find the best parameters for simulations of isolated molecules and ions with organic and biological interest. Our research is going in that direction.

\section{Acknowledgement}

We thank ANR DynBioReact (Grant No. ANR-14-CE06-0029-01) and CNRS program INFINITI (project ASTROCOL) for support. We also thank William L. Hase for useful discussions and for providing us the modified version of Venus and input for microcanonical simulations. 


\section{Appendix: $\mathrm{CH}_{4}$ PES}

Here we summarize the functional form and actual parameters used in the $\mathrm{CH}_{4}$ potential energy surface used, which originate from DHS potential ${ }^{47}$ with subsequent modifications. ${ }^{48,49}$ The total potential has the expression:

$$
\begin{aligned}
V_{\text {total }} & =\sum_{i=1}^{4} D_{i}\left[1-e^{B_{i}\left(r_{i}-r_{i}^{0}\right)}\right]^{2}+\frac{1}{2} \sum_{i=1}^{3} \sum_{j>i}^{4} f_{i j}\left(\theta_{i j}-\theta_{i j}^{0}\right)^{2}+\sum_{i=1}^{3} \sum_{j>i}^{4} g_{i j}\left(\theta_{i j}-\theta_{i j}^{0}\right)^{3} \\
& +\sum_{i=1}^{3} \sum_{j>i}^{4} h_{i j}\left(\theta_{i j}-\theta_{i j}^{0}\right)^{4}+\sum_{i=1}^{4} f_{\Delta i} \sum_{\substack{j=1 \\
j \neq i}}^{4} \Delta_{i j}^{2}+\sum_{i=1}^{4} h_{\Delta i} \sum_{\substack{j=1 \\
j \neq i}}^{4} \Delta_{i j}^{4}+g_{n 4} C_{4}
\end{aligned}
$$

The first term is a sum of Morse potential for the $\mathrm{C}-\mathrm{H}$ bonds, with the following parameters: $D_{i}=109.460 \mathrm{kcal} / \mathrm{mol}, B_{i}=1.933 \AA^{-1}$ and $r_{i}^{0}=1.090 \AA$. The angular terms (second, third and fourth) have a complex form which can be divided in three parts: (i) quadratic, cubic and quartic angular potentials; (ii) out-of-plane quadratic and quartic potentials and (iii) non-diagonal cubic potential. Before expressing these terms, the equilibrium angle value, $\theta_{i j}^{0}$, should take into account of the differences between equilibrium and asymptotic values. At this end the following expression is used:

$$
\theta_{i j}^{0}=\theta_{i j}^{t}+\left(\theta_{i j}^{t}-\theta_{i j}^{(1)}\right)\left[S_{\theta}^{(1)}\left(r_{i}\right) S_{\theta}^{(1)}\left(r_{j}\right)-1\right]+\left(\theta_{i j}^{t}-\theta_{i j}^{(2)}\right)\left[S_{\theta}^{(2)}\left(r_{k}\right) S_{\theta}^{(2)}\left(r_{l}\right)-1\right]
$$

with $\theta_{i j}^{t}=109.4712^{\circ}, \theta_{i j}^{(1)}=90^{\circ}$ and $\theta_{i j}^{(2)}=120^{\circ} . S_{\theta}^{(1)}$ and $S_{\theta}^{(2)}$ are two switching functions to smoothly move from equilibrium $\mathrm{CH}_{4}$ geometry to dissociating $\mathrm{CH}_{3}+\mathrm{H}$ system with correct $\mathrm{CH}_{3}$ geometries at infinite distance between $\mathrm{CH}_{3}$ and $\mathrm{H}$. They have the following forms: 


$$
\begin{gathered}
S_{\theta}^{(1)}=1.0-\tanh \left[\alpha_{P}\left(r_{i}-R_{0}\right)\left(e^{\beta_{P}\left(r_{i}-\gamma_{P}\right)^{3}}+1\right)\right] \\
S_{\theta}^{(2)}=1.0-\tanh \left[\alpha_{T}\left(r_{i}-R_{0}\right)\left(e^{\beta_{T}\left(r_{i}-\gamma_{T}\right)^{3}}+1\right)\right]
\end{gathered}
$$

with $R_{0}=1.086 \AA, \alpha_{P}=0.224147141 \AA^{-1}, \beta_{P}=-0.990736407 \cdot 10^{-5} \AA^{-3}, \gamma_{P}=0.238179398 \cdot 10^{2} \AA$, $\alpha_{T}=0.330879271 \AA^{-1}, \beta_{T}=-0.12408758 \cdot 10^{-2} \AA^{-3}$ and $\gamma_{T}=0.880601978 \cdot 10^{1} \AA$.

The force constants are also modified by the switching functions:

$$
\begin{aligned}
f_{i j} & =f_{i j}^{0}\left[S_{f}^{(1)}\left(r_{i}\right) S_{f}^{(1)}\left(r_{j}\right)-1\right]+\left(f_{i j}^{0}-f_{i j}^{(2)}\right)\left[S_{f}^{(2)}\left(r_{k}\right) S_{f}^{(2)}\left(r_{l}\right)-1\right] S_{f}^{(1)}\left(r_{i}\right) S_{f}^{(1)}\left(r_{j}\right) \\
g_{i j} & =g_{i j}^{0}\left[S_{g}^{(1)}\left(r_{i}\right) S_{g}^{(1)}\left(r_{j}\right)-1\right]+\left(g_{i j}^{0}-g_{i j}^{(2)}\right)\left[S_{g}^{(2)}\left(r_{k}\right) S_{g}^{(2)}\left(r_{l}\right)-1\right] S_{g}^{(1)}\left(r_{i}\right) S_{g}^{(1)}\left(r_{j}\right) \\
h_{i j} & =h_{i j}^{0}\left[S_{h}^{(1)}\left(r_{i}\right) S_{h}^{(1)}\left(r_{j}\right)-1\right]+\left(h_{i j}^{0}-h_{i j}^{(2)}\right)\left[S_{h}^{(2)}\left(r_{k}\right) S_{h}^{(2)}\left(r_{l}\right)-1\right] S_{h}^{(1)}\left(r_{i}\right) S_{h}^{(1)}\left(r_{j}\right)
\end{aligned}
$$

where: $f_{i j}^{0}=0.5938, f_{i j}^{(2)}=0.4543, g_{i j}^{0}=-0.0996, g_{i j}^{(2)}=-0.1232, h_{i j}^{0}=0.0996$ and $h_{i j}^{(2)}=-0.0101$. And the switching functions are:

$$
\begin{aligned}
S_{f}^{(1)} & =1.0-\tanh \left[\alpha_{1}\left(r-R_{0}\right)\left(r-\beta_{1}\right)^{\gamma_{1}}\right] \\
S_{f}^{(2)} & =1.0-\tanh \left[\alpha_{2}\left(r-R_{0}\right)\left(r-\beta_{2}\right)^{\gamma_{2}}\right] \\
S_{g}^{(1)} & =1.0-\tanh \left[\alpha_{4}\left(r-R_{0}\right)\left(r-\beta_{4}\right)^{\gamma_{4}}\right] \\
S_{g}^{(2)} & =S_{f}^{(2)} \\
S_{h}^{(1)} & =S_{g}^{(1)} \\
S_{h}^{(2)} & =S_{g}^{(2)}
\end{aligned}
$$

Then the out-of-plane potential (fifth and sixth terms in Eq. 16) has the force constants modified by the switching functions: 


$$
\begin{aligned}
f_{\Delta i} & =\left(1-S_{\Delta}\left(r_{i}\right)\right) S_{\Delta}\left(r_{j}\right) S_{\Delta}\left(r_{k}\right) S_{\Delta}\left(r_{l}\right) f_{\Delta i}^{(1)} \\
h_{\Delta i} & =\left(1-S_{\Delta}\left(r_{i}\right)\right) S_{\Delta}\left(r_{j}\right) S_{\Delta}\left(r_{k}\right) S_{\Delta}\left(r_{l}\right) h_{\Delta i}^{(1)} \\
S_{\Delta} & =1.0-\tanh \left[\alpha_{3}\left(r-R_{0}\right)\left(r-\beta_{3}\right)^{\gamma_{3}}\right]
\end{aligned}
$$

with $f_{\Delta i}^{(1)}=0.0436, h_{\Delta i}^{(1)}=0.0854, \alpha_{3}=1.4191474 \cdot 10^{-1}, \beta_{3}=-3.0684503 \cdot 10^{-1}$ and $\gamma_{3}=2.0$. The out-of-plane angle is defined as:

$$
\Delta_{i j}=\cos ^{-1}\left[\vec{N} \cdot \vec{r}_{j} /\left|\vec{r}_{i}\right|\right]-\theta_{i j}^{0}
$$

where

$$
\vec{N}=\frac{\left(\vec{r}_{k}-\vec{r}_{j}\right) \wedge\left(\vec{r}_{i}-\vec{r}_{j}\right)}{\left|\left(\vec{r}_{k}-\vec{r}_{j}\right) \wedge\left(\vec{r}_{i}-\vec{r}_{j}\right)\right|}
$$

where $i=1,2,3,4$ ( 5 is the $\mathrm{C}$ atom).

Finally, the non-diagonal cubic potential (last term in Eq. 16) is composed by force constants $g_{n i}$ which are all zero except $g_{n 4}$ :

$$
g_{n 4}=\left[\prod_{j=1}^{4} S_{g}^{n}\left(r_{j}\right)\right] g_{n 4}^{C H 4}
$$

with $g_{n 4}^{C H 4}=0.2242$ and $S_{g}^{n}=S_{\Delta}$ and

$$
\begin{aligned}
C_{4} & =\left(\theta_{12}-\theta_{12}^{0}\right)\left(\theta_{13}-\theta_{13}^{0}\right)\left(\theta_{23}-\theta_{23}^{0}\right)+\left(\theta_{14}-\theta_{14}^{0}\right)\left(\theta_{12}-\theta_{12}^{0}\right)\left(\theta_{24}-\theta_{24}^{0}\right) \\
& +\left(\theta_{14}-\theta_{14}^{0}\right)\left(\theta_{13}-\theta_{13}^{0}\right)\left(\theta_{34}-\theta_{34}^{0}\right)+\left(\theta_{24}-\theta_{24}^{0}\right)\left(\theta_{23}-\theta_{23}^{0}\right)\left(\theta_{34}-\theta_{34}^{0}\right)
\end{aligned}
$$




\section{References}

(1) Baer, T.; Hase, W. L. Unimolecular reaction dynamics: theory and experiments; Oxford University Press, New York, 1996.

(2) Bunker, D. L. Monte Carlo Calculation of Triatomic Dissociation Rates. I. $\mathrm{N}_{2}$ O. J. Chem. Phys. 1962, 37, 393-403.

(3) Forst, W. Theory of unimolecular reactions; Academic Press, New York, 1973.

(4) Wardlaw, D. M.; Marcus, R. A. On the statistical theory of unimolecular processes. Adv. Chem. Phys. 1988, 70, 231-263.

(5) Klots, C. E. Reformulation of the quasi-equilibrium theory of ionic fragmentation. $J$. Phys. Chem. 1971, 75, 1526-1532.

(6) Klots, C. E. Quasi-equilibrium theory of ionic fragmentation: further considerations. Z. Naturforsch. 1972, 27a, 553-561.

(7) Klots, C. E. Thermochemical and kinetic information from metastable decompositions of ions. J. Chem. Phys. 1973, 58, 5364.

(8) Lourderaj, U.; McAfee, J. L.; Hase, W. L. Potential Energy Surface and Unimolecular Dynamics of Stretched n-Butane. J. Chem. Phys. 2008, 129, 094701.

(9) Yang, L.; Sun, R.; Hase, W. L. Use of Direct Dynamics Simulations to Determine Unimolecular Reaction Paths and Arrhenius Parameters for Large Molecules. J. Chem. Theory Comput. 2012, \%, 3478-3483.

(10) Ma, X.; Paul, A. K.; Hase, W. L. Chemical Dynamics Simulations of Benzene Dimer Dissociation. J. Phys. Chem. A 2015, 119, 6631-6640. 
(11) Kolakkandy, S.; Paul, A. K.; Pratihar, S.; Kohale, S. C.; Barnes, G. L.; Wang, H.; Hase, W. L. Energy and Temperature Dependent Dissociation of the $\mathrm{Na}^{+}$(benzene) ${ }_{1,2}$ Clusters. Importance of Anharmonicity. J. Chem. Phys. 2015, 142, 044306.

(12) Song, K.; Spezia, R. Theoretical Mass Spectrometry; De Gruyter, Berlin, 2018.

(13) Spezia, R.; Salpin, J.-Y.; Gaigeot, M.-P.; Hase, W.; Song, K. Protonated Urea CollisionInduced Dissociation. Comparison of Experiments and Chemical Dynamics Simulations. J. Phys. Chem. A 2009, 113, 13853-13862.

(14) Spezia, R.; Cimas, A.; Gaigeot, M.-P.; Salpin, J.-Y.; Song, K.; Hase, W. Collision Induced Dissociation of Doubly-charged Ions: Coulomb Explosion vs Neutral Loss in $[\mathrm{Ca}(\text { urea })]^{2+}$ Gas Phase Unimolecular Reactivity via Chemical Dynamics Simulations. Phys. Chem. Chem. Phys. 2012, 14, 11724-11736.

(15) Martin-Somer, A.; Yanez, M.; Gaigeot, M.-P.; Spezia, R. Unimolecular Fragmentation Induced By Low Energy Collision: Statistically Or Dynamically Driven? J. Phys. Chem. A 2014, 118, 10882-10893.

(16) Spezia, R.; Martin-Somer, A.; Macaluso, V.; Homayoon, Z.; Pratihar, S.; Hase, W. L. Unimolecular Dissociation of Peptides: Statistical vs Non-Statistical Fragmentation Mechanisms and Time Scales. Faraday Discuss. 2016, 195, 599-618.

(17) Martin-Somer, A.; Martens, J.; Grzetic, J.; Hase, W. L.; Oomens, J.; Spezia, R. Unimolecular fragmentation of deprotonated diproline $\left[\mathrm{PrO}_{2}-\mathrm{H}\right]^{-}$studied by chemical dynamics simulations and IRMPD spectroscopy. J. Phys. Chem. A 2018, 122, 2612-2625.

(18) Homayoon, Z.; Pratihar, S.; Dratz, E.; Snider, R.; Spezia, R.; Barnes, G.; Macaluso, V.; Martin-Somer, A.; Hase, W. L. Model Simulations of the Thermal Dissociation of the $\operatorname{TIK}\left(\mathrm{H}^{+}\right)_{2}$ tripeptide. Mechanisms and Kinetic Parameters. J. Phys. Chem. A 2016, 120, 8211-8227. 
(19) Spezia, R.; Martens, J.; Oomens, J.; Song, K. Collision-induced dissociation pathways of protonated $\mathrm{Gly}_{2} \mathrm{NH}_{2}$ and $\mathrm{Gly}_{3} \mathrm{NH}_{2}$ in the short time-scale limit by chemical dynamics and ion spectroscopy. Int. J. Mass Spectrom. 2015, 388, 40-52.

(20) Spezia, R.; Lee, S. B.; Cho, A.; Song, K. Collision Induced Dissociation Mechanisms of Protonated Penta- and Octa-Glycine as Revealed by Chemical Dynamics Simulations. Int. J. Mass Spectrom. 2015, 392, 125-138.

(21) Ortiz, D.; Salpin, J.-Y.; Song, K.; Spezia, R. Galactose 6-sulfate collision induced dissociation using QM+MM chemical dynamics simulations and ESI-MS/MS experiments. Int. J. Mass Spectrom. 2014, 358, 25-35.

(22) Rossich Molina, E.; Eizaguirre, A.; Haldys, V.; Urban, D.; Doisneau, G.; Bourdreux, Y.; J.-M.Beau,; Salpin, J.-Y.; R.Spezia, Characterization of protonated model disaccharides from tandem mass spectrometry and chemical dynamics simulations. ChemPhysChem 2017, 18, 2812-2823.

(23) Craig, I. R.; Manolopoulos, D. E. Quantum statistics and classical mechanics: Real time correlation functions from ring polymer molecular dynamics. J. Chem. Phys. 2004, 121, 3368.

(24) Cao, J.; Voth, G. A. The formulation of quantum statistical mechanics based on the Feynman path centroid density. II. Dynamical properties. J. Chem. Phys. 1994, 100, 5106-5117.

(25) Habershon, S.; Manolopoulos, D. E. Zero point energy leakage in condensed phase dynamics: An assessment of quantum simulation methods for liquid water. J. Chem. Phys. 2009, 131, 244518.

(26) Perez de Tudela, R.; Aoiz, F. J.; Suleimanov, Y. V.; Manolopoulos, D. E. Chemical reaction rates from ring polymer molecular dynamics: Zero point energy conservation in $\mathrm{Mu}+\mathrm{H}_{2}$ to $\mathrm{MuH}+$ H. J. Phys. Chem. Lett. 2012, 3, 493-497. 
(27) Feynman, R. P.; Hibbs, A. R. Quantum mechanics and path integrals; McGraw-Hill, New York, 1965.

(28) Feynman, R. P. Statistical mechanics; Addison-Wesley, Reading, MA, 1972.

(29) Berne, B. J.; Thirumalai, D. On the Simulation of Quantum Systems: Path Integral Methods. Ann. Rev. Phys. Chem. 1986, 37, 401-424.

(30) Perez, A.; Tuckerman, M. E.; Muser, M. H. A comparative study of the centroid and ring-polymer molecular dynamics methods for approximating quantum time correlation functions from path integrals. J. Chem. Phys. 2009, 130, 184105.

(31) Kay, K. G. Semiclassical Initial Value Treatments of Atoms and Molecules. Annu. Rev. Phys. Chem. 2005, 56, 255-280.

(32) Herman, M. F.; Kluk, E. A semiclassical justification for the use of non-spreading wavepackets in dynamics calculations. Chem. Phys. 1984, 91, 27-34.

(33) Buchholz, M.; Fallacara, E.; Gottwald, F.; Ceotto, M.; Grossmann, F.; Ivanov, S. D. Herman-Kluk propagator is free from zero-point energy leakage. Chem. Phys. 2018, $515,231-235$.

(34) Bowman, J. M.; Gazdy, B.; Sun, Q. A method to constrain vibrational energy in quasiclassical trajectory calculations. J. Chem. Phys. 1989, 91, 2859.

(35) Czakó, G.; Kaledin, A. L.; Bowman, J. M. A practical method to avoid zero-point leak in molecular dynamics calculations: Application to the water dimer. J. Chem. Phys. 2010, 132, 164103.

(36) Lim, K. F.; McCormack, D. A. The conservation of quantum zero-point energies in classical trajectory simulations. J. Chem. Phys. 1995, 102, 1705.

(37) McCormack, D. A.; Lim, K. F. The zero-point energy problem in classical trajectory simulations at dissociation threshold. J. Chem. Phys. 1997, 106, 572. 
(38) Dammak, H.; Chalopin, Y.; Laroche, M.; Hayoun, M.; Greffet, J.-J. Quantum Thermal Bath for Molecular Dynamics Simulation. Phys. Rev. Lett. 2009, 103, 190601.

(39) Dammak, H.; Antoshchenkova, E.; Hayoun, M.; Finocchi, F. Isotope effects in lithium hydride and lithium deuteride crystals by molecular dynamics simulations. J. Phys. Cond. Matt. 2012, 24, 435402.

(40) Calvo, F.; Van-Oanh, N.; Parneix, P.; Falvo, C. Vibrational spectra of polyatomic molecules assisted by quantum thermal baths. Phys. Chem. Chem. Phys. 2012, 14, 10503.

(41) Savin, A. V.; Kosevich, Y. A.; Cantarero, A. Semiquantum molecular dynamics simulation of thermal properties and heat transport in low-dimensional nanostructures. Phys. Rev. B 2012, 86, 064305 .

(42) Basire, M.; Borgis, D.; Vuilleumier, R. Computing Wigner distributions and time correlation functions using the quantum thermal bath method: application to proton transfer spectroscopy. Phys. Chem. Chem. Phys. 2013, 15, 12591.

(43) Bronstein, Y.; Depondt, P.; Finocchi, F.; Saitta, A. M. Quantum-driven phase transition in ice described via an efficient Langevin approach. Phys. Rev. B 2014, 89, 214101.

(44) Bronstein, Y.; Depondt, P.; Finocchi, F. Thermal and nuclear quantum effects in the hydrogen bond dynamical symmetrization phase transition of -AlOOH. Eur. J. Mineral. 2017, 29, 385-395.

(45) Paul, A.; Hase, W. L. Zero-Point Energy Constraint for Unimolecular Dissociation Reactions. Giving Trajectories Multiple Chances To Dissociate Correctly. J. Phys. Chem. A 2016, 120, 372-378. 
(46) Miller, W. H.; Hase, W. L.; Darling, C. L. A simple model for correcting the zero point energy problem in classical trajectory simulations of polyatomic molecules. J. Chem. Phys. 1989, 91, 2863.

(47) Duchovic, R. J.; Hase, W. L.; Schlegel, H. B. Analytic Function for the H $+\mathrm{CH}_{3}=$ $\mathrm{CH}_{4}$ Potential Energy Surface. J. Phys. Chem. 1984, 88, 1339.

(48) Hase, W. L.; Mondro, S. L.; Duchovic, R. J.; Hirst, D. M. Thermal Rate Constant for $\mathrm{H}+\mathrm{CH}_{3} \rightarrow \mathrm{CH}_{4}$ Recombination. 3. Comparison of Experiment and Canonical Variational Transition State Theory. J. Am. Chem. Soc. 1987, 109, 2916-2922.

(49) Hu, X.; Hase, W. L.; Pirraglia, T. Vectorization of the General Monte Carlo Classical Trajectory Program VENUS. J. Comput. Chem. 1991, 12, 1014-1024.

(50) Warmbier, R.; Schneider, P.; Sharma, A. R.; Braams, B. J.; Bowman, J. M.; Hauschildt, P. H. Ab Initio Modeling of Molecular IR Spectra of Astrophysical Interest: Application to $\mathrm{CH}_{4}$. Astron. Astrophys. 2009, 495, 655-661.

(51) Conte, R.; Houston, P. L.; Bowman, J. M. Trajectory and Model Studies of Collisions of Highly Excited Methane with Water Using and ab Initio Potential. J. Phys. Chem. A 2015, 119, 12304-12317.

(52) Callen, H. B.; Welton, T. A. Irreversibility and Generalized Noise. Phys. Rev. 1951, 83,34 .

(53) Chalopin, Y.; Dammak, H.; Laroche, M.; Hayoun, M.; Greffet, J. J. Radiative heat transfer from a black body to dielectric nanoparticles. Phys. Rev. B 2011, 84, 224301.

(54) Brieuc, F.; Bronstein, Y.; Dammak, H.; Depondt, P.; Finocchi, F.; Hayoun, M. ZeroPoint Energy Leakage in Quantum Thermal Bath Molecular Dynamics Simulations. J. Chem. Theory Comput. 2016, 12, 5688-5697. 
(55) Hase, W. L.; Duchovic, R. J.; Hu, X.; Komornicki, A.; Lim, K. F.; Lu, D.-H.; Peslherbe, G. H.; Swamy, K. N.; Linde, S. R. V.; Varandas, A. et al. VENUS. A General Chemical Dynamics Computer Program. QCPE Bull. 1996, 16, 671.

(56) Mangaud, E.; Huppert, S.; Pl, T.; Depondt, P.; Bonella, S.; Finocchi, F. The FluctuationDissipation Theorem as a Diagnosis and Cure for Zero-Point Energy Leakage in Quantum Thermal Bath Simulations. J. Chem. Theory Comput. 2019, 15, 2863-2880.

(57) Johnston, H.; Birks, J. Activation Energies for the Dissociation of Diatomic Molecules Are Less than the Bond Dissociation Energies. Acc. Chem. Res. 1972, 5, 327-335.

(58) Truhlar, D. Interpretation of the Activation Energy. J. Chem. Edu. 1978, 55, 309-311.

(59) Song, K.; Meroueh, O.; Hase, W. L. Dynamics of $\mathrm{Cr}(\mathrm{CO})_{6}^{+}$collisions with hydrogenated surfaces. J. Chem. Phys. 2003, 118, 2893-2902.

(60) Troe, J. Specific rate constants k(E,J) for unimolecular bond fissions. J. Chem. Phys. 1983, 79, 6017.

(61) Troe, J. Simplified models for anharmonic numbers and densities of vibrational states. I. Application to $\mathrm{NO}_{2}$ and $\mathrm{H}_{3}^{+}$. Chem. Phys. 1995, 190, 381-392. 